\title{
Hypertensive Disorders During Pregnancy in Gestational Diabetic Women
}

Authors

Affiliation

Key words

- gestational diabetes

- hypertension

- pregnancy

- risk factors

- complications

- outcomes received $\quad 06.11 .2007$

first decision 06.11.2007

accepted 23.11.2007

Bibliography

DOI 10.1055/s-2007-1004524

Exp Clin Endocrinol Diabetes

2008; 116: 329-332

(C). A. Barth Verlag in

Georg Thieme Verlag KG

Stuttgart - New York

ISSN 0947-7349

Correspondence

Prof. C. Savona-Ventura

"NorthWynds" 7 Antonio

Zammit Street

GHR2040 Gharghur

Malta

charles.savona-ventura@

um.edu.mt
C. Savona-Ventura, S. Grima

Department of Obstetrics-Gynaecology, Department of Health, Malta

\begin{abstract}
$\nabla$

Gestational diabetes is often compounded by hypertensive disease of pregnancy. Women diagnosed as suffering from gestational diabetes were subdivided into two groups: those found to be suffering from some form of hypertension during their pregnancy [ $\mathrm{n}=78]$; and those who did not develop hypertension [ $\mathrm{n}=345]$. The maternal characteristics and perinatal outcome
\end{abstract}

\section{Introduction}

$\nabla$

Women with gestational diabetes have been shown to have a significantly 2.5 -times higher risk of having a pregnancy complicated by hypertensive disease of mixed aetiology when compared to women with normal carbohydrate metabolism [1]. This observation is not at all surprising since gestational diabetes has been shown to be a harbinger of later onset metabolic syndrome. The appearance of hypertensive disease should further compound the clinical situation with increased stress being placed on the mother and the placento-fetal unit giving rise to increased maternal and fetal morto-morbidity. The present study attempts to quantify the clinical effect of hypertensive disease on GDM pregnancies.

\section{Material and Methods $\nabla$}

Women confirmed as suffering from gestational diabetes mellitus [GDM] defined as a blood glucose 2-hour post-75g sugar load of $8.6 \mathrm{mmol} / \mathrm{l}$ or more were subdivided into two groups. The first group was composed of $78 \mathrm{GDM}$ individuals who during pregnancy were found to suffer from hypertension as defined by the WHO criteria; the second group was composed of $345 \mathrm{GDM}$ individuals whose blood pressure remained per- of the two groups were statistically correlated. The results show that obesity was the only statistically significant correlate, and that obstetric outcome more often required planned delivery by induction of labour or caesarean section. The infant was more likely to be delivered prematurely. While the perinatal morbidity did not show any significant differences, this was only because of the more active intervention policy in these women. sistently in the normal range. Cases of multiple births in GDM women were excluded from the study. The patients' clinical data was reviewed to identify a number of maternal biological characteristics [age, pre-pregnancy BMI, parity, miscarriage historyl, antenatal obstetric problems [antepartum haemorrhage, use of insulin in treatment of GDM], intrapartum problems [onset of labour, type of delivery], and infant outcome parameters [birth weight, gestation at delivery, conditions causing neonatal morbidity, and perinatal mortality]. The two groups were analysed statistically using the student $t$ test and the chi square test as appropriate. A probability value of $<0.05$ was taken to signify statistical significance.

\section{Results}

\section{$\nabla$}

Women suffering from gestational diabetes appeared to be more likely to develop hypertensive disease if they were elderly, obese, and primiparous or multiparous. However, the only statistically significant correlate was an elevated Body Mass Index (Table 1). The development of hypertensive disease in GDM women did not appear to increase the risks of antepartum haemorrhage. The GDM appeared to more likely require insulin treatment in its management in 
Table 1 Maternal Characteristics and complications

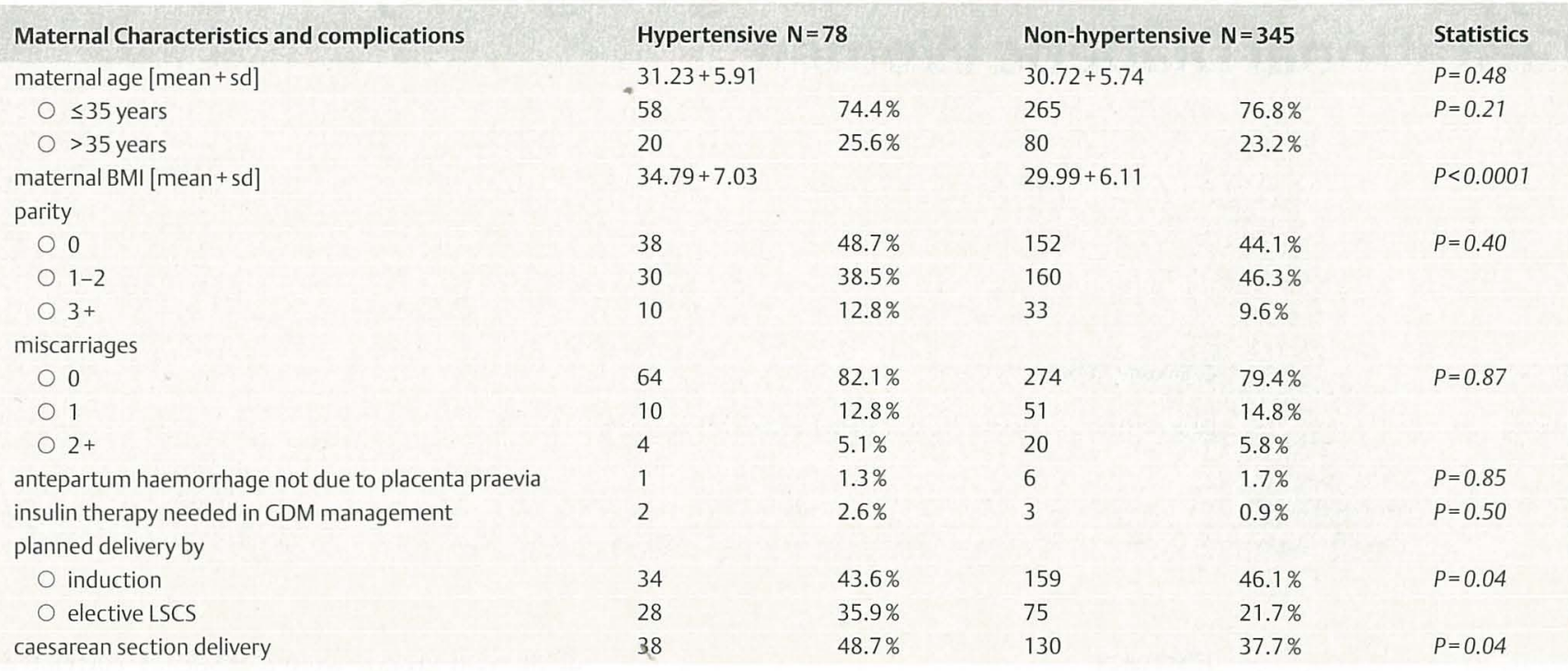

Table 2 Infant Characteristics and complications

\begin{tabular}{|c|c|c|c|c|c|}
\hline Infant Characteristics and complications & \multicolumn{2}{|c|}{ Hypertensive $\mathrm{N}=78$} & \multicolumn{2}{|c|}{ Non-hypertensive $\mathrm{N}=345$} & Statistics \\
\hline birth weight [mean+sd] & \multicolumn{2}{|c|}{$3394+600.31$} & \multicolumn{2}{|c|}{$3456.59+562.18$} & $P=0.38$ \\
\hline $0<2500$ grams & 4 & $5.2 \%$ & 14 & $4.1 \%$ & $P=0.87$ \\
\hline $2500-3999 \mathrm{gm}$ & 65 & $83.3 \%$ & 286 & $82.9 \%$ & \\
\hline $0>4000$ grams & 9 & $11.5 \%$ & 45 & $13.0 \%$ & \\
\hline \multicolumn{6}{|l|}{ prematurity } \\
\hline $0<34$ weeks & 7 & $9.0 \%$ & 8 & $2.3 \%$ & $P=0.03$ \\
\hline $35-36$ weeks & 9 & $11.5 \%$ & 25 & $7.3 \%$ & \\
\hline apgar score $=<7 @ 5 \mathrm{~min}$ & 1 & $1.3 \%$ & 10 & $2.9 \%$ & $P=0.68$ \\
\hline respiratory distress syndrome & 8 & $10.3 \%$ & 18 & $5.2 \%$ & $P=0.17$ \\
\hline shoulder dystocia & 3 & $3.9 \%$ & 4 & $1.2 \%$ & $P=0.24$ \\
\hline postnatal seizures & 0 & - & 2 & $0.6 \%$ & $P=0.81$ \\
\hline perinatal mortality & 0 & - & 3 & $0.9 \%$ & $P=0.94$ \\
\hline
\end{tabular}

the presence of hypertension though the difference did not show statistical significance. The development of hypertension in GDM women was statistically associated with a higher rate of obstetric intervention as evident by planned deliveries through elective caesarean section or induction of labour [79.5\% in GDM+ hypertension vs $67.8 \%$ in GDM-hypertension; $p=0.0393$ ]. There was also a statistically significant higher rate of elective and emergency caesarean delivery required in those GDM women with hypertension [48.7\% vs. 37.7\%; p=0.0441] (Table 1).

The higher obstetric intervention rates in GDM women with hypertension results in earlier delivery statistically increasing the rate of premature delivery from $9.6 \%$ in GDM women without hypertension to $20.5 \%$ in GDM women suffering also from hypertension. The increase in prematurity rate also showed a tendency towards greater rates of respiratory distress syndrome and low birth weight, though these differences did not reach statistical significance (Table 2 ).

There did not appear in the hypertensive group to be any statistically significant differences in birth weigh averages when correlated to maternal age, BMI, parity, and infant gender. In contrast there did appear to be a statistically significant correlation to maternal BMI and parity in the non-hypertensive group of mothers. There were no correlations in birth weight averages between the hypertensive and non-hypertensive groups (Table 3). There appeared to be a greater predisposition to $>95^{\text {th }}$ percentile birth weights by gestation as assessed for the Maltese population irrespective of the presence or absence of hypertension ( $\mathbf{F i g} \mathbf{1}$ ).

\section{Discussion}

$\nabla$

The pregnant state though the influence of the hormones produced by the placental induces a series of physiological changes in the mother aimed at facilitating nutrient transfer to the developing fetus. This physiological adjustment is clearly evident and well established in the changes induced by the placental hormones in carbohydrate metabolism. The gestational increase in insulin resistance in the peripheral tissues results in frank gestational diabetes in those previously insulin resistant but metabolically compensated individuals. The prevalence of gestational diabetes would therefore be expected to be significantly higher than that in the reproductive age female population. In the Maltese female population in the reproductive age group, the prevalence of newly diagnosed diabetes mellitus and impaired glucose tolerance had been estimated by epidemiological studies conducted in the early 1980 s to be $1.8 \%$ [2]. During the same period, the prevalence of gestational diabetes according to the ADA criteria of a 2-hour post-glucose load of $8.6 \mathrm{mmol} / \mathrm{l}$ or more had been estimated at $5.9 \%$ [3]. Gestational diabetes has been further shown to be a strong indicator for the 


\begin{tabular}{|c|c|c|c|}
\hline Birth Weight correlates [mean $+\mathrm{sd}$ ] & Hypertensive $\mathrm{N}=78$ & Non-hypertensive $\mathrm{N}=345$ & Statistics \\
\hline \multicolumn{4}{|l|}{ maternal age } \\
\hline $0<=35$ years & $3369+590[58]$ & $3459+555[265]$ & $P=0.27$ \\
\hline $0>35$ years & $\begin{array}{l}3468+639.6[20] \\
p=0.53\end{array}$ & $\begin{array}{l}3447.39+589.36[80] \\
p=0.87\end{array}$ & $P=0.89$ \\
\hline \multicolumn{4}{|l|}{ maternal pre-pregnancy BMI } \\
\hline $0<30 \mathrm{~kg} / \mathrm{m}^{2}$ & $3240.24+737.55[21]$ & $3354.69+500.69$ & $P=0.35$ \\
\hline $0>30 \mathrm{~kg} / \mathrm{m}^{2}$ & $\begin{array}{l}3451.4+537.61[57] \\
p=0.17\end{array}$ & $\begin{array}{l}{[170] 3555.57+601.11} \\
{[175] p=0.0009}\end{array}$ & $P=0.25$ \\
\hline \multicolumn{4}{|l|}{ parity } \\
\hline $\mathrm{OPO}$ & $3273+704[38]$ & $3361+564[152]$ & $P=0.42$ \\
\hline $\mathrm{P} 1+$ & $\begin{array}{l}3510.25+461.52[40] \\
p=0.08\end{array}$ & $\begin{array}{l}3531.75+550.51[193] \\
p=0.005\end{array}$ & $P=0.82$ \\
\hline \multicolumn{4}{|l|}{ infant gender } \\
\hline male & $3462+514[46]$ & $3490+550[178]$ & $P=0.76$ \\
\hline female & $\begin{array}{l}3298.13+703.99[32] \\
p=0.24\end{array}$ & $\begin{array}{l}3421.16+574.1[167] \\
p=0.26\end{array}$ & $P=0.29$ \\
\hline
\end{tabular}

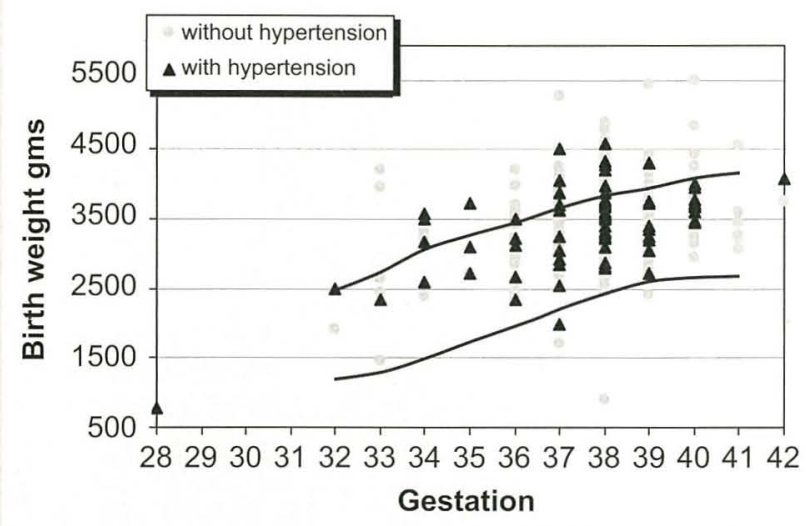

Fig. 1 Birth weights by gestation and population birth weight $5 \%$ and $95 \%$ centiles.

development of adult-onset diabetes in many populations, including the Maltese [4].

Patients with GDM have been shown to have a different diastolic function profile, suggesting a mild degree of diastolic abnormality which in some individuals persisted into the postpartum period. The interrelationship between GDM and diastolic abnormalities reflects the presence of endothelial dysfunction which may be mediated by reduced levels of the vasodilator Angiotensin 1-7 $[5,6]$. The prevalence of hypertension in the nonpregnant Maltese female population of reproductive age in the early 1980 s was estimated at $5.9 \%$ [2]. The rate of hypertensive disease in pregnancy was during the same period reported as $6.8 \%$ [7], a figure that has been approximately maintained in the period 2000-2004 [8]. Maltese women with gestational diabetes have been reported as having a greater incidence of hypertensive disease reaching a rate of about $16.5 \%$ [1]. The apparent association between hypertensive disease and gestational diabetes suggests that there might be an interrelationship between these two conditions in the form of an underlying metabolic syndrome. The present study has further linked the development of hypertension in women with GDM to obesity, which itself has been shown to be contributory feature to the development of the metabolic syndrome [9]. The statistically unconfirmed apparent association in this study of hypertension in GDM women to advanced age and increased recourse to insulin therapy may suggest a more advanced deterioration in the metabolic state of the hypertensive woman. Increasing maternal age has been repeatedly shown to be a correlate to increasing maternal morbidity from both hypertension and diabetes [7]. It has been shown that undue increase in insulin resistance during pregnancy may manifest both pregnancy-induced hypertension and gestational diabetes [10].

Hypertensive diseases of pregnancy are the most common serious medical disorders seen by obstetricians. These disorders constitute a range of disorders with different aetiologies and pathology. They are generally classified into clinical categories without reference to aetiology. Broadly, they may be sub-divided into pre-existing conditions, pre-eclampsia syndrome, and gestational hypertension. The latter group includes a mixture of patients who either develop pre-eclampsia syndrome without proteinuria or have a latent underlying essential hypertension which becomes evident during pregnancy [11]. All the hypertensive disorders have long been associated with higher rates of maternal and fetal morto-morbidity; however the pre-eclampsia syndrome gives a greater contribution towards adverse events. No attempt have been made in the present study to differentiate between the various categories of hypertension, but pre-eclampsia syndrome was reported in about $6 \%$ of all the hypertensive cases; the remainder being either cases of chronic or gestational hypertension. This would explain why the present study failed to demonstrate any particular increase in morbidity further than that caused by an earlier obstetric intervention.

The development of hypertensive disease in women with gestational diabetes increases the morbidity to both the mother and the neonatal. The clinical management thus often requires intervention to prematurely deliver the child with the consequences of a greater recourse to operative abdominal delivery with the associated increased morbidity of this surgery. The premature neonate is also placed a greater risks of morbidity since the care required by these infants has to be more intensive and complicated. While the incidence of respiratory distress seemed to be slightly increased in neonates born to hypertensive GDM mother, there did not appear to be any significant deleterious effects. Gestational diabetes appears to have a greater risk of being complicated with hypertensive disease. This is not surprising in the light of the known association between essential hypertension and type 2 diabetes mellitus outside pregnancy. The most likely underlying pathophysiology is latent insulin resistance in 
the woman which in the face of the physiological stresses caused by pregnancy exhibits itself by the development of both gestational diabetes and hypertension. This is more likely to occur in the presence of maternal obesity. While the perinatal morbidity in hypertensive GDM women does not appear to be significantly affected when compared to non-hypertensive ones, strict antenatal vigilance of GDM women remains essential since the perinatal morbidity is only kept at a low level by timely intervention.

\section{References}

1 Savona-Ventura C, Ellul A, Chircop M. The outcome of diabetic pregnancies in Malta. International Journal of Gynecology \& Obstetrics 2003; 82: $217-218$

2 Katona G, Aganovic I, Vuskan V, Skrabalo Z. The National Diabetes Programme in Malta - Final report Phases I\&II. WHO.NCD/OND/DIAB/83.2. WHO, Geneva 1983

3 Savona-Ventura C, Schranz AG, Chazan B. The clinical significance of gestational impaired glucose tolerance in the Maltese population. Arch Perinatal Med 1997; 3: 55-64 [the prevalence rates have been reviewed to reflect the new diagnostic criteria]

4 Schranz AG, Savona-Ventura C. Long-term significance of mild Gestational Diabetes Mellitus - A Longitudinal Study. Exper Clin Endocrinol Diabetes 2002; 110: 219-222
5 Freire CM, Nunes Mdo C, Barbosa MM, Longo JR, Nogueira AI, Diniz SS, Machado LJ, Oliveira AR de. Gestational diabetes: a condition of early diastolic abnormalities in young women. J Am Soc Echocardiogr 2006; 19 (10): 1251-1256

6 Nogueira AI, Souza Santos RA, Simoes E Silva AC, Cabral AC, Vieira RL, Drumond TC, Machado LJ, Freire CM, Ribeiro-Oliveira Jr A. The pregnancy-induced increase of plasma angiotensin-(1-7) is blunted in gestational diabetes. Regul Pept 2007; 141 (1-3): 55-60

7 Savona-Ventura C, Micallef I, Grech ES. Trends in maternal mortality and morbidity in the Maltese Islands. Proc.: E.S.S.A.R. Vth Annual meeting, Malta, September 1987. Government Press, Malta, 1988; 109-118

8 Janulova L National Obstetric Information System (NOIS), Malta, Annual Report 2000-2004. DHI, Malta, 2001-2005 [annual publications - available at http://www.sahha.gov.mt/pages.aspx?page=121]

9 Savona-Ventura C, Gatt M. Short-term obstetric outcomes in obese Maltese women. International Journal of Diabetes and Metabolism 2006; 14 (2): 88-91

10 Yang $X L$, Lau JT. Undue increase in insulin resistance during pregnancy may manifest pregnancy-induced hypertension and gestational diabetes. Horm Metab Res 2004; 36 (10): 655-661

11 WHO Study Group. The hypertensive disorders of pregnancy. Technical Report Series 758, WHO, Geneva 1987 